\title{
Singlet Oxygen Generation From Porphyrin-Functionalized Hexahedral Polysilicon Microparticles
}

\section{Gordon Bruce, ${ }^{a}$ Mario Samperi, ${ }^{b, c}$ David B. Amabilino, ${ }^{b, c}$ Marta Duch, ${ }^{d}$ José A. Plaza ${ }^{d}$ and Lluïsa Pérez-García ${ }^{* a}$}

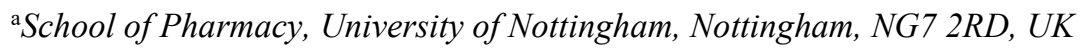 \\ ${ }^{\mathrm{b}}$ School of Chemistry, University of Nottingham, University Park, NG7 2RD, UK \\ ${ }^{\mathrm{c}}$ The GSK Carbon Neutral Laboratories for Sustainable Chemistry, University of Nottingham, Triumph Road, \\ Nottingham NG7 2TU, UK \\ ${ }^{\mathrm{d} I n s t i t u t o}$ de Microelectrónica de Barcelona, IMB-CNM (CSIC), C/ dels Til·lers, Campus UAB, Cerdanyola del Vallès, \\ Barcelona 08193 , Spain
}

Received date (to be automatically inserted after your manuscript is submitted)

Accepted date (to be automatically inserted after your manuscript is accepted)

\begin{abstract}
The generation of singlet oxygen (SO), primarily by using a combination of light and photosensitizers in the presence of the dissolved gas, finds applications in both chemistry and medicine. The efficiency of its formation can be enhanced by immobilization of the photosensitizers. In this work, we have explored the covalent functionalization in suspension of hexahedral slab-like polysilicon microparticles ( $\mu \mathbf{P}$, with a largest dimension of three microns) with a model photosensitizer, 5-(4isothiocyanatophenyl)-10,15,20-(triphenyl)porphyrin (ITC-P), and evaluated the singlet oxygen generation of this photosensitiser in solution and after immobilization (ITC-P- $\mu \mathbf{P})$ in suspension. The SO detection experiment on the functionalised microparticles was performed using a hydrogel as the matrix supporting the microparticles (to avoid their settling), and revealed that ITC-P- $\boldsymbol{\mu P}$ in suspension are capable of generating SO more efficiently than the free ITC-P in solution.
\end{abstract}

KEYWORDS: hybrid material, porphyrin immobilization, monolayer, microparticles in suspension, photoexcitation, singlet oxygen production, hydrogel

*Correspondence to: Lluïsa Pérez-García, School of Pharmacy, University of Nottingham, Nottingham, NG7 2RD, UK, email: Lluisa.PerezGarcia1@nottingham.ac.uk, tel. +44 (0) 1157486256. 


\section{INTRODUCTION}

Hybrid materials obtained by the combination of micro- and nanotechnology are considered as extremely relevant to science and technology, in particular for applications in biomedical science, catalysis and waste treatment [1-3]. Advances in micro- and nano-fabrication methods have provided new means of fabricating micro-and nano-devices for drug and gene delivery [4-6], tissue engineering [7], biosensors [8,9] and diagnostic systems, for proteins[8], DNA microarrays[10] and microfluidic circuits for biochemical sample preparation [11].

The achievement of (bio)chemical functionality relies on the use of different materials as substrates for the formation of self-assembled monolayers (SAMs) [12], gold [3,13] and silicon [9,14] being the most widely used and studied, because of the ease of using thiol and silane connectors, respectively, to be attached onto the corresponding surfaces to form well-organized SAMs and also because the substrates are biocompatible and chemically stable materials. Some of the most important challenges concerning SAMs usage are to select a methodology to obtain a well-organized SAM, to find a simple method to characterize and quantify the monolayer [15] and, more significantly, to be able to correlate chemical synthesis, characterization and function of the new material. Besides the SAMs have been synthetized to produce biosensors, super hydrophilic/hydrophobic surfaces, charged surfaces or to endow substrates of several properties [16,17]. So it provides great potential in surface design of monolayers for bioactive coating for biomedical devices such as drug delivery and sensor systems [18-21].

Our interest in these systems stems from their potential as platforms to perform varying functions, a main one being the generation of singlet oxygen (SO), for various reasons: The chemistry of SO plays a key role in many bio-chemical processes, and it is central to many new phenomena both in chemistry and biology and generating applications in many fields ranging from organic synthetic chemistry [22] to biomedical science [23]. Its generation is most frequently achieved induced by using a combination of light and photosensitizers, often with a high SO quantum yields [24,25]. In particular, porphyrins are effective photosensitisers, that have been increasingly studied on account of their numerous applications in different areas such as photochemistry, molecular recognition [26], sensors [27], molecular machines [28] or in photodynamic therapy (PDT) [29]. Different vehicles such as gold nanoparticles [30], silica nanoparticles [31], and polymeric microparticles[32] containing porphyrins to use in PDT have been reported. The enhanced generation of SO from immobilised photosensitisers has been observed in polymerised zinc porphyrazine nanospheres [32]. On the other hand, SO generated from immobilized porphyrins and phthalocyanines can be used to degrade polutants [33]: By way of example, a porphyrin immobilised on silica gel was shown to degrade metoprolol via reaction with SO through different kinetics (exponential rather than pseudo first order for a solution analogue) but to a lesser degree than the dissolved photosensitiser [34]. When coated onto nanoparticles, the SO generation can be impaired compared with freely dissolved porphyrins [35], and therefore the platform and linking are clearly determining in the properties of the hybrid materials.

In recent years we have worked in the (bio)chemical functionalization of microfabricated silicon based microparticles and studied their applications in biology. Thus, we have reported on the adhesion of lectin-functionalized encoded microparticles to the zona pellucida of embryos for tagging purposes [8,9,14], on the internalization of silicon oxide microparticles in HeLa cells as intracellular pH sensors [36], as well as on the interacion of multi-material microparticles with different types of cell lines [3]. On the other hand, we have also explored the phototoxicity of photosensitiserfunctionalized iron oxide [37] and gold nanoparticles [38-40] as potential carriers of use in PDT [41]. All our previous findings suggest that polysilicon is an excellent material to prepare hybrid materials through its chemical modification because it is robust, easy to funcionalize, biocompatible, and a common material in the semiconductor industry, that allows a wide variety of designs for the functional devices [42]. For this reason, in this work we explore the covalent immobilization of a selected photosensitiser on microfabricated polysilicon microparticles aiming to assess its potential as SO generator. The compoud 5-(4-isothiocyanatophenyl)-10,15,20-(triphenyl)porphyrin (ITC-P) was chosen as 
photosensitiser, and also a thiourea porphyrin derivative was synthesized and envisaged as a solution model of the immobilization. ITC-P was immobilized on previously amino-funcionalized polysilicon microparticles in suspension, and characterization of the functionalization was carried out using fluorescence spectroscopy and microscopy. The efficiency of the new hybrid material for generating SO was assessed for the photosensitizer both in solution and after immobilization in the silicon devices.

\section{RESULTS AND DISCUSSION}

\section{Microfabrication of polysilicon microparticles $(\mu \mathrm{P})$.}

The fabrication process of the polysilicon microparticles $(\mu \mathrm{P})$ is shown schematically in Fig. 1, and is based on a method previously described [43]. Briefly, a four-inch (100) p-type silicon wafer is used as a substrate. Then, a $1 \mu \mathrm{m}$ thick silicon oxide layer that acts as a sacrificial layer was deposited onto the silicon wafer by Plasma Enhanced Chemical Vapor deposition (Fig. 1b). A $0.5 \mu \mathrm{m}$ polysilicon device layer was deposited by chemical vapor deposition (Fig. 1c). A photoresist layer was spun on the wafer followed by a photographic step (Fig. 1d and e). The chips were patterned by a polysilicon dry etching (Fig. 1f). The photoresist was removed by plasma etching (Fig. 1g) and finally the chips were released from the wafer by etching the sacrificial silicon oxide layer in hydrofluoric acid (HF) 50\% (Fig. 1h) and suspended in ethanol using ultrasound. The chips have to be filtered using a $5 \mu \mathrm{m}$ filter rating and centrifuged at 14000 rpm for 5 min. More than 150 million devices for each four-inch wafer are obtained with controlled and reproducible shapes and dimensions. Fig. 1 also shows optical microscopy images of the $\mu \mathbf{P}$ before (Fig. 1i) and after release (Fig. 1j) from the wafer.

$<$ Fig. $1>$

The dimensions of the free $\mu \mathbf{P}$ are $3 \times 3 \times 0.5 \mu \mathrm{m}^{3}$, as represented in Fig. 2a, making them easily identifiable even under an optical microscope. Fig. $2 b$ shows a SEM image of these $\boldsymbol{\mu P}$, which were the substrates used for chemical functionalization in suspension.

$<$ Fig. 2>

\section{Synthesis and characterisation of the porphyrin conjugate TUEE-P}

The two porphyrin derivatives used in this work are shown in Scheme 1. 5-(4-Isothiocyanatophenyl)-10,15,20(triphenyl)porphyrin (ITC-P) is commercially available and was selected to be immobilized on $\boldsymbol{\mu P}$, whereas its thiourea derivative TUEE-P was chosen as a model, because of its higher solubility and stability in solution. For its synthesis, ITC-P was made to react with 2-(2-aminoethoxy)ethanol at room temperature for $2 \mathrm{~h}$ to give TUEE-P, as shown in Scheme S1 (Supporting Information). The yield of the reaction was low mainly because of the difficulty of its separation by column chromatography, but the reaction was not further optimized. The characterization of the conjugate TUEE-P can be found in the Supporting Information (Fig. S1-S2).

$<$ Scheme 1 $>$ 


\section{Immobilization of ITC-P on polysilicon microparticles $(\mu \mathrm{P})$}

The self-assembled monolayer (SAM) of ITC-P on the surface of $\mu \mathbf{P}$ was prepared using covalent bonding, because of their strength and stability, and the formation of SAMs relies on silicon chemistry procedures, based on the formation of covalent $\mathrm{Si}-\mathrm{O}$ bonds. The silane reagent incorporates the desired alkyl chain, to assist in a good packing of the SAM, with a functional group appropriate for the posterior modification and linkage to the porphyrin ITC-P. In this work, the selected spacer consisted of an eleven carbon atom alkyl chain, and an amino group was chosen as the terminal group to form thiourea type bonds stable in biological conditions. Thus, the immobilization of ITC-P follows a 3 step protocol based on a) surface activation by piranha solution followed by basic treatment, b) reaction with 11aminoundecyltriethoxysilane (AUTES) in ethanol for $2 \mathrm{~h}$, and c) reaction of the amino functionalized surface with ITC$\mathbf{P}$ in acetone overnight, to finally form ITC-P- $\mu \mathbf{P}$, where the porphyrin is linked to the surface through a thiourea bond (Scheme 1). The concentration of both the silane linker and ICT-P may influence the outcome of the functionalization of ITC-P- $\mu$ P. The choice of concentration is based on our previous experience on the functionalization of similar polysilicon materials [8], with an excess of reagents being used to promote optimal coverage of the surface. The main difficulty associated to the functionalization results from the fact of the $\mu \mathbf{P}$ being in suspension, which requires gentle but continous shaking to avoid aggregation or breaking the particles and ensure the uniformity of the functionalization.

Also, purification by centrifugation is associated with loss of some $\mu \mathbf{P}$ at each chemical step. For this reason, the number of particles was counted manually using a Neubauer chamber, and we estimate that throughout the functionalization process shown in Scheme 1 the particle loss was ca.90\%, i.e., a typical sample starts the process with $1,500,000 \mu \mathbf{P}$ and by the end of the process contains 150,000 ITC-P- $\mu \mathbf{P}$.

The amount of porphyrin immobilized on ITC-P- $\mu \mathbf{P}$ was determined indirectly by UV-vis absorption spectroscopy after obtaining a calibration curve for ITC-P in acetone $\left(\varepsilon=440,000 \mathrm{M}^{-1} \cdot \mathrm{cm}^{-1}\right)$, which is shown in Fig. S3 in the Supporting Information. Thus, surface funtionalization was estimated by calculating the difference between the initial concentration of ITC-P in the solution used for the chemical functionalization and the amount remaining in the supernatant. Also, a calibration curve for TUEE-P in acetone $\left(\varepsilon=342,000 \mathrm{M}^{-1} \cdot \mathrm{cm}^{-1}\right)$ was obtained, which is shown in Fig. S4 in the Supporting Information.

The presence of the porphyrin on ITC-P- $\boldsymbol{\mu P}$, indicating their successful immobilization, was assessed by fluorescence microscopy and spectroscopy. The functionalized microparticles ITC-P- $\mu$ P show bright fluorescence observed due to the presence of the porphyrins on their surfaces. Fluorescence emission spectra of the model TUEE-P and also ITC-P- $\mu$ P are shown in Fig. S5 in the Supporting Information. Both spectra were recorded following excitation at $415 \mathrm{~nm}$ in acetone solution and suspension, respectively, and their comparison indicates that there is no a significant difference upon immobilization, since the reduction in the fluorescence intensity associated to ITC-P- $\mu \mathbf{P}$ is related to the smaller content of the porphyrin in the sample $(0.62 \mu \mathrm{M})$, compared to the free porphyrin TUEE-P in solution $(9 \mu \mathrm{M})$.

Furthermore the fluorescence microscope images of ITC-P- $\boldsymbol{\mu P}$ show homogeneous coverage of the chromophore on the silicon surface on each particle, as can be seen in Fig. 2c. To analyse further the homogenity of the functionalization, the intensity of the fluorescence was measured for 20 representative particles after defining four cross sections, as illustrated in Fig. 2d, and Fig. 2e shows the fluorescence's intensity profile of one particles alongside the 4 cross section respect the lateral dimension of the surface of the particle. The results indicate homogeneous fluorescence intensity and no significant differences were observed between the microparticles with immobilized porphyrins ITC-P- $\boldsymbol{\mu P}$. However, a striking characteristic is that the perimeter of the flat-lying objects is clearly brighter than the centre of the hexahedral 
slabs, indicating, therefore, a higher concentration of the fluorophore in these regions at this orientation with respect to the objective. It is believed that the reason for this effect is that at any point on the surface of the slab, in principle, one would expect to observe fluorescence from a maximum of two chromophores, one on the top side of the particle and the other on the bottom side (assuming the polysilicon of this thickness to be partially transparent). On the edges of the particle, however, the whole area can be functionalised and rather than having two layers of porphyrins there are expected to be at least several dozen (as the edges are $500 \mathrm{~nm}$ deep), even allowing for partial coverage. This fact can be appreciated from the schematic drawing of the functionalised particle shown in Fig. $2 \mathrm{f}$. The observed relative intensity of the edge and face areas varies slightly according to the focal plane, but all the profiles of fluorescence intensity across the particles show a predominance of fluorescence at the edges.

\section{Singlet oxygen generation}

Singlet oxygen generation was examined using the acid-functionalised anthracene probe 9,10-anthracenedylbis(methylene)dimalonic acid (ADMA) that has been employed for this purpose previously with other chromophores [39]. In the presence of SO, ADMA is converted into an endoperoxide (see Fig. S6 in the Supporting Information), leading to a decrease in the fluorescence emission of the molecule due to photobleaching. The emission decay of ADMA can be easily followed using fluorescence spectroscopy (Fig. 3). To study the SO production, after a control irradiating only ADMA (Fig. 3a), the photosensitiser was irradiated in the presence of ADMA with a solar simulator (xenon lamp with $495 \mathrm{~nm}$ filter) as the light source. Initially, we aimed to compare the decay of ITC-P either in acetone solution or in suspension when immobilized on the microparticles ITC-P- $\mu \mathbf{P}$, and the results are shown in Fig. S7 in the Supporting Information. Fluorescence emission spectra were recorded every $10 \mathrm{~min}$, in the range of 390-550 nm, and the SO production was determined by the decrease of the emission intensity of the ADMA. The porphyrin ITC-P shows an effective SO generation upon irradiation using a solar simulator, since it was observed that after 1 hour the decay of the fluorescence of ADMA in the presence of ITC-P was significant (ca. 50\%). There is a short induction period (ca 10 min), after which the fluorescence of the anthracene probe molecule decays steadily. Instead, when the porphyrin functionalised microparticles ITC-P- $\mu$ P were suspended in acetone under the same conditions as ITC-P with the ADMA and light was used to generate SO, a rapid generation of SO was observed by quenching of the fluorescence of the anthracene derivative (ca. 20\%), but this value held steady after just $10 \mathrm{~min}$. We interpreted this effect as being a result of possible binding of the acid group of the ADMA to either the porphyrin base or more likely to unreacted amine groups on the surface of the microparticles. However, when the experiment was performed in the presence of a base $(\mathrm{NaOH}) \mathrm{no}$ difference was observed (data not shown).

However, an additional factor that hinders a ready observation of the generation of SO by the microparticles is that they settle in non-viscous solutions, i.e. they settle to the bottom of the cuvette being used for the measurement. This effect was also seen when the particles were suspended in water with a solution of the sodium salt of the anthracene derivative.

Therefore, we sought a medium where the particles could be immobilised and yet still generate SO that would diffuse to react with ADMA. We chose a supramolecular gelator that forms a hydrogel when an ethanol solution of the small molecule is mixed with an aqueous solution. In this case, the sodium salt of ADMA in water was mixed with an ethanol solution of gelator, and a transparent gel formed with the particles held in the matrix of nanometre fibres in the colloidal suspension. A solution in acetone of ITC-P has the same macroscopic appearance than when ITC-P- $\mu$ P is incorporated within the gel (see Fig. S8 in the Supporting Information).

Fig. 3 shows the time-dependent decay of the ADMA emission spectrum following irradiation of ADMA, ITC-P, TUEE-P and ITC-P- $\mu$ P. Control samples containing ADMA either in acetone solution (Fig. 3a) or incorporated in the 
gel (Fig. 3b) without any porphyrin, irradiated under the same conditions as described above (see also Experimental Section) shows a negligible decay in the ADMA fluorescence emission. This result confirms that SO was produced by the porphyrin derivatives, alone or loaded in the $\mu \mathbf{P}$, upon irradiation, and it also proves that the chemical integrity of ADMA is mantained both in solution and the gel. It was observed that the decay of the emission of ADMA was high upon irradiation of acetone solutions of ITC-P (Fig. 3c) and TUEE-P (Fig. 3d), as well as of ITC-P- $\mu$ P within the gel (Fig. 3e). Indeed, irradiation of this sample ITC-P- $\mu$ P with the solar simulator caused a very significant generation of SO, evidenced by the decrease of the fluorescence of the ADMA. The sample was stable even in the light beam for several minutes, although because of opacity arising in the gel, the mixture was heated and the gel reformed between certain fluorescence measurements and further irradiation. Therefore the gel has a role of immobilising the microparticles, but at the same time allowing solvent and oxygen to diffuse through the sample. It is established that gels contain liquid that can move through the network of fibres generated by the gelator. Therefore, this kind of system is a useful one for immobilising small particles so that their photoreactivity can be studied.

$<$ Fig. 3 $>$

A summary of the progressive decay of the ADMA emission band at $\lambda=430 \mathrm{~nm}$ upon irradiation of all samples is shown in Fig. 4a, indicating the percentage of the ADMA that had been converted to the endoperoxide. After $1 \mathrm{~h}$ following irradiation of the samples, the levels of ADMA decay for ITC-P in acetone, TUEE-P in acetone, and ITC-P- $\mu$ P in the gel were $48 \%, 48 \%$, and $65 \%$, respectively, indicating the much faster and more efficient generation of SO by the porphyrin immobilized in ITC-P- $\boldsymbol{\mu P}$. Further irradiation up to $2 \mathrm{~h}$ induces levels of decay for TUEE-P in acetone and ITC-P- $\mu \mathbf{P}$ in the gel of $70 \%$ and $80 \%$, respectively, indicating that SO is generated at a slower rate for ITC-P- $\mu \mathbf{P}$. All the progressive values shown in Fig. 4a are much higher than those previously reported for similar porphyrins immobilized in gold nano- or microparticles in aqueous solutions [38, 44], and they are specially remarkable in the case of ITC-P- $\mu \mathbf{P}$, given the fact that the hydrogel dispersion contains a high content of water.

$<$ Fig. $4>$

To be able to compare the SO production of the different samples, the maximum rate of ADMA photobleaching was normalized using the corresponding concentration of the photosensitizer and following Eq. (1).

$$
\frac{\left(\% \mathrm{IF}_{431} \text { at } t=0 \mathrm{~min}\right)-\left(\% \mathrm{IF}_{431} \text { at } t=60 \mathrm{~min}\right)}{60 \mathrm{~min} \times[\text { porphyrin }](\mu \mathrm{M})}
$$

Equation used to calculate the maximum rate of ADMA photobleaching.

The calculated \% rates of ADMA photobleaching for the three analyzed samples are shown in Fig. 4b. ITC-P- $\mu$ P in the gel (\% rate of ADMA photobleaching of $2.6 \% \mathrm{FI} /(\mathrm{min} . \mathrm{mM}))$ produce SO in a much more efficient manner than either ITC-P or TUEE-P in acetone $(0.1 \% \mathrm{FI} /(\min . \mathrm{mM}))$, something extremely significant considering the much lower concentration of porphyrin needed to quantify the effect. This last observation also clearly indicates the enhancement in SO production induced by the immobilization of the porphyrin onto the microparticles (ITC-P- $\mu$ P) in an aqueous environment.

The reaction of the SO with ADMA follows a first order decay in the case of the homogeneous solution of TUEE-P (see Fig. S9a in the Supporting Information) as a result of the excess of the reagent. In contrast, the microparticles in the gel follow a second order kinetics (see Fig. S9b in the Supporting Information), that has also been observed in silica gel hybrid systems,[34] although in the present case the conversion and rate of reaction are far greater. 
In conclusion, we have shown that the immobilization of ITC-P in hexahedral polysilicon microparticles in suspension proceeds with homogeneous coverage of the chromophore on the silicon surface on each particle, ensuring the homogeneity of the suspensions of functionalized microparticles, as shown by fluorescence microscopy. The excitation and emission properties of the porphyrin do not seem to be affected upon immobilization, as indicated by fluorescence spectroscopy. More importantly, the immobilization of the photosensitiser on polysilicon microparticles at a micromolar concentration improves the SO generation with respect to more concentrated solutions of the parent photosensitising agent. The difficulty of measuring the SO generation of the functionalized microparticles ITC-P- $\mu \mathbf{P}$ in suspension is overcome by incorporating them in a hydrogel that acts as a framework to immobilise the particles while enabling difusion of oxygen and reagent whereby the photoreaction proceeds extremely effectively, as well as providing an aqueous environment where the SO is produced with high efficiency. The enhancement in SO generation induced by this type of hybrid material makes it an attractive candidate to be used in different applications when efficient SO production is required.

\section{EXPERIMENTAL}

\section{General Methods}

Solvents. All solvents were purchased and dried through prepacked dessecant columns: dichloromethane (DCM) from Sigma-Aldrich, chloroform, methanol and ethanol from Fisher, and acetone from VWR.

Commercially available reagents. 2-(2-Aminoethoxy)ethanol, ammonium hydroxide (20\%), 9,10-anthracenedylbis(methylene)dimalonic acid (ADMA), hydrogen peroxide, sodium sulfate anhydrous and sulfuric acid (96\%) were purchased from Sigma-Aldrich. 11-Aminoundecyltriethoxysilane (AUTES) was purchased from Alfa Aesar, and 5-(4isothiocyanatophenyl)-10,15,20-(triphenyl)porphyrin (ITC-P) from Porphychem. Water used in the functionalization experiments was HPLC grade produced by a Milli-Q plus system from Millipore (Milli-Q water).

Melting points were measured using a Stuart SMP20 melting point apparatus. ${ }^{1} \mathrm{H}$ NMR spectra were recorded using a Bruker AV400 (400 MHz) using $\mathrm{CDCl}_{3}$ as solvent, and the chemical shifts are expressed in parts per million (ppm) relative to the central peak of the solvent. IR spectra were recorded using an ALPHA II Platinum ATR single reflection diamond ATR module (Bruker). Thin layer chromatography (TLC) was performed on Merck silica gel plates coated with $\mathrm{F}_{254}$ fluorescent indicator. Column chromatography was carried out on silica gel 60 (Aldrich, technical grade, 230-400 mesh). UV-Vis absorbance spectroscopy was measured in acetone using a Cary $5000 \mathrm{UV}-\mathrm{Vis}$ spectrophotometer (Agilent), using quart cuvettes with a $1 \mathrm{~cm}$ path length. Brightfield and Fluorescence images of the particles were acquired using a Nikon Eclipse Ti-U fluorescence microscope. $\lambda_{\mathrm{Ex}}=405 \mathrm{~nm} \lambda_{\mathrm{Em}}=>620 \mathrm{~nm}$ (40x objective, 1 second excitation). Fluorescence spectroscopy was measured using a FLS 980 spectrometer (Edinburgh Instruments) equipped with a front face sample holder and longpass filter at $600 \mathrm{~nm}$. The fluorescence excitation and emission spectra were recorded in right angle using quartz cuvettes with a $0.2 \mathrm{~cm}$ path length.

\section{Synthesis and characterization}

\section{Preparation of TUEE-Porphyrin conjugate (TUEE-P).}

A solution of 2-(2-aminoethoxy)ethanol solution $(5 \mathrm{mg}, 0.05 \mathrm{mmol})$ in DCM (2 mL) was added to a solution of 5-(4isothiocyanatophenyl)-10,15,20-(triphenyl)porphyrin (ITC-P) $(20 \mathrm{mg}, 0.03 \mathrm{mmol})$ in DCM (10 mL), and the reaction mixture left to stir under argon atmosphere at room temperature for 2 hours. After this time, the reaction mixture was extracted with water $(3 \times 10 \mathrm{~mL})$, the organic phase dried over sodium sulfate anhydrous, and the solvent evaporated 
under reduced pressure. The resulting residue was purified using silica gel chromatography with chloroform/methanol (90:10) as the eluent, to give TUEE-P (3.8 mg, 16.3\%), mp $209{ }^{\circ} \mathrm{C}$. UV-Vis (Acetone): $\lambda_{\max }(\mathrm{nm}) 415,512,544,599$. ${ }^{1} \mathrm{H}$ NMR $(400 \mathrm{MHz}$, Chloroform- $d$ ) : $\delta$ 8.78-8.90 (m, 8H, pyrrole- $H), 8.25-8.17\left(\mathrm{~d}, 2 \mathrm{H}, J=7.8 \mathrm{~Hz}, \mathrm{H}_{\mathrm{Ar} 2}, 6^{\circ}\right), 8.17-8.08$ (m, 6H, $\left.\mathrm{H}_{\mathrm{Ar} 2,6}\right), 7.76-7.63\left(\mathrm{~m}, 9 \mathrm{H}, \mathrm{H}_{\mathrm{Ar} 3,4,5}\right), 7.57$ (d, 2H, J = 7.8 Hz, H $\left.\mathrm{Ar}{ }^{\prime}, 5^{\prime}\right), 3.99$ (m, 2H, $\left.\mathrm{CH}_{2}-\mathrm{OH}\right), 3.81$ (t, $2 \mathrm{H}, \mathrm{CH}_{2}-$ O), $3.70\left(\mathrm{~m}, 2 \mathrm{H}, \mathrm{CH}_{2}-\mathrm{NH}\right), 3.63-3.56\left(\mathrm{t}, 2 \mathrm{H}, \mathrm{CH}_{2}-\mathrm{O}\right),-2.85$ (s, 2H, pyrrole- $\mathrm{NH}$ ). IR (solid) $v_{\max } 3313,3051,3023,2922$, $1700,1595,1472,1441,1348,1348,1123,1071,965,797,724,699 \mathrm{~cm}^{-1}$.

\section{Chemical Functionalization of Microparticles (ITC-P- $\mu$ P)}

The polysilicon microparticles $(\mu \mathrm{P})$ used in this study were prepared as described elsewhere [43].

Surface activation. Polysilicon microparticles (ca. 1.6 million particles) were treated with piranha solution $(500 \mu \mathrm{L})$ $\mathrm{H}_{2} \mathrm{SO}_{4} / \mathrm{H}_{2} \mathrm{O}_{2}$ (7:3) for 1 hour and then washed with milliQ water $(3 \times 700 \mu \mathrm{L})$ using centrifugation (5700 RPM, 15 minutes). The particles were then treated with a basic solution $(500 \mu \mathrm{L}) \mathrm{H}_{2} \mathrm{O} / \mathrm{NH}_{4} \mathrm{OH} / \mathrm{H}_{2} \mathrm{O}_{2}(5: 1: 1)$ and then washed with milliQ water $(3 \times 700 \mu \mathrm{L})$ and once with absolute ethanol $(700 \mu \mathrm{L})$ using centrifugation after each step (5700 RPM, 15 minutes).

Surface functionalization. A 1\% solution of 11-aminoundecyltriethoxysilane (AUTES) in absolute ethanol (500 $\mu \mathrm{L})$ was added to the particles and gentle shaking was mantained for 2 hours using a vortex. Subsequently the particles were washed with ethanol $(3 \times 700 \mu \mathrm{L})$ and once with acetone $(700 \mu \mathrm{L})$ using centrifugation (5700 RPM, 15 minutes).

A solution of ITC-P $(10 \mu \mathrm{g}, 0.015 \mu \mathrm{mol})$ in acetone $(500 \mu \mathrm{L})$ was prepared and the $\mu \mathrm{P}$ immersed in this solution overnight. The particles were then washed with acetone $(5 \times 700 \mu \mathrm{L})$ and chloroform $(2 \times 700 \mu \mathrm{L})$ using centrifugation (5700 RPM, 15 minutes). The washings were retained for analysis.

Calculation of amount of ITC-P immobilized on (ITC-P- $\boldsymbol{\mu P})$. A calibration curve for ITC-P in acetone was obtained (see Fig. S4 in Supporting Information), consisting of solutions of ITC-P in acetone with known concentrations (0.3, 1, 2, 3, 4, $5 \mu \mathrm{M}$ ), and the extinction coefficient of the ITC-P in acetone was calculated. The supernatant from chemical functionalization of the $\mu \mathrm{P}$ was dried and then dissolved in acetone $(2.5 \mathrm{~mL})$. The absorbance of this solution at $415 \mathrm{~nm}$ was measured using UV-Vis absorption spectroscopy and fitted in the calibration curve of ITC-P to obtain the concentration in the supernatant. The amount of immobilized ITC-P on the particles was calculated by subtracting the amount of porphyrin in the washings from the initial amount of porphyrin in the ITC-P solution used for the chemical functionalization.

\section{Microparticles Characterisation}

Counting $\mu \mathrm{P}$ in suspension. Particles were counted manually by adding $10 \mu \mathrm{L}$ of particle suspension to a Neubauer chamber and counting the particles in each of the four quadrants. An average of each of the quadrants was then used to calculate the total number of particles.

Fluorescence analysis. Images of fluorescent particles acquired using a Nikon Eclipse Ti-U fluorescence microscope were analysed using ImageJ software. 4 cross sections of each particle (20 particles) were analysed and relative values of the fluorescence were obtained relative to the intensity of the non-functionalized background.

\section{Singlet oxygen generation}

Singlet oxygen production of ITC-P and TUEE-P was measured by monitoring the fluorescence decay of 9,10anthracenedyl-bis(methylene)dimalonic acid (ADMA) in acetone solution in the presence of ITC-P or TUEE-P $(9 \mu \mathrm{M})$. The molar ratio of ADMA and porphyrin was kept constant (2.2:1) for each sample. A $600 \mu \mathrm{L}$ cuvette containing the 
sample was irradiated using a xenon lamp with $495 \mathrm{~nm}$ filter at $0.3 \mathrm{sun}\left(30 \mathrm{~mW} / \mathrm{cm}^{2}\right)$ for 2 hours and the fluorescence emission spectra of ADMA recorded between 390 and $550 \mathrm{~nm}\left(\lambda_{\mathrm{ex}}=380 \mathrm{~nm}\right)$ at regular intervals using a FLS 980 spectrometer (Edinburgh Instruments).

For measurement of SO generation from microparticles a gelator, a 1,3:2,4-Dibenzylidene-D-sorbitol derivative [45] was used to keep the microparticles suspended throughout the irradiation period. A $15 \mathrm{mM}$ solution of DBS-derivative (300 $\mu \mathrm{L}, 4.5 \mu \mathrm{mol}$ ) in absolute ethanol was added to a $600 \mu \mathrm{L}$ cuvette containing 157,000 microparticles in $172 \mu \mathrm{L}$ of MilliQ water. To this, $7.5 \mu \mathrm{L}$ of ADMA $(80 \mu \mathrm{M})$ stock solution in $0.3 \mathrm{mM} \mathrm{NaOH}$ was added and the volume made up to 600 $\mu \mathrm{L}$ with $120 \mu \mathrm{L}$ absolute ethanol. The sample was then irradiated as previously described and emission spectra recorded. To ensure that there was no optical interference of the gelator with the fluorescence measurements the gel was heated until clear before each measurement was taken. A control for this procedure was obtained in the absence of particles to ensure no effect of the gelator on fluorescence measurements.

\section{Acknowledgements.}

L.P-G thanks the University of Nottingham for a Anne McLaren Research Fellowship. G.B. thanks EPSRC Grant EP/L01646X and GSK for a predoctoral grant. This work was supported by by EU ERDF (FEDER) funds and the Spanish Government grant TEC2017-85059-C3-1-2-R, as well as the Engineering and Physical Research Council grant EP/R004072/1. We warmly thank Glyssa Dizon for providing the gelator.

Supporting Information. A full list of one Scheme (Scheme S1) and nine Figures (Fig. S1-S9) are given in the supplementary material. This material is available at http://www.u.bourgogne.fr/jpp/

\section{REFERENCES}

[1] Lim CT, Han J, Guck J, Espinosa H, Med. Biol. Eng. Comput. 2010; 48: 941-943.

[2] Caldorera-Moore M, Peppas NA, Adv. Drug Deliv Rev. 2010; 61: 1391-1401.

[3] Patiño T, Soriano J, Amirthalingam E, Durán S, González-Campo A, Duch M, Ibáñez E, Barrios L, Plaza J, PérezGarcía L, Nogués C, Nanoscale 2016; 8: 8773-8783.

[4] Maye MM, Nykypanchuk D, Van Der Lelie D, Gang O, Small 2007; 3: 1678-1682.

[5] Guo S, Wang E, Acc. Chem. Res. 2011; 44: 491-500.

[6] Staples M, Daniel K, Cima MJ, Langer R, Pharm. Res. 2006; 23: 847-863.

[7] Andersson H, van den Berg A, Lab Chip 2004; 4: 98-103.

[8] Penon O, Novo S, Durán S, Ibañez E, Nogués C, Samitier J, Duch M, Plaza JA, Pérez-García L, Bioconjugate Chem. 2012; 23: 2392-2402.

[9] Novo S, Penon O, Barrios L, Nogués C, Santaló J, Durán S, Gómez-Matínez R, , Samitier J, Plaza JA, Pérez-García L, Ibáñez E, Hum. Reprod. 2013; 28: 1519-1527.

[10] Chiou EP-Y, Tsutsui H, J. Lab. Autom. 2013; 18: 425-426.

[11] Zuvin M, Mansur N, Birol SZ, Trabzon L, Say1 Yazgan A, Microsyst. Technol., 2016; 22: 645-652.

[12] Amabilino DB, Supramolecular Chemistry at Surfaces, RSC, Cambridge, 2016.

[13] Durán S, Duch M, Patiño T, Torres A, Penon O, Gómez-Martínez R, Barrios L, Esteve J, Nogués C, Pérez-García L, Plaza JA, Sensors Actuators B Chem. 2015; 209: 212-224.

[14] Penon O, Siapkas D, Novo S, Durán S, Oncins G, Errachid A, Barrios L, Nogués C, Duch M, Plaza JA, PérezGarcía L, Colloids Surf. B Biointerfaces 2014: 116: 104-113.

[15] Böhmler J, Ploux L, Ball V, Anselme K, Ponche A, J. Phys. Chem. C. 2011; 115: 11102-11111. 
[16] Samanta D, Sarkar A, Chem. Soc. Rev. 2011; 40: 2567-2592.

[17] Sakeye M, Smaištt JH, Langmuir 2012; 28: 16941-16950.

[18] Shalek AK, Robinson JT, Karp ES, Lee JS, Ahn D-R, Yoon M-H, Sutton A, Jorgolli M, Gertner RS, Gujral TS, MacBeath G, Yang EG, Park H, Proc. Natl. Acad. Sci. U.S.A. 2010; 107: 1870-1875.

[19] Hilt JZ, Peppas NA, Int. J. Pharm. 2005; 306: 15-23.

[20] Siqueira JR, Caseli L, Crespilho FN, Zucolotto V, Oliveira ON, Biosens. Bioelectron. 2010; 25: 1254-1263.

[21] Cheng D, Shang F, Ratner J, Bioconjugate Chem. 2012; 100: 130-134.

[22] Ghogare AA, Greer A, Chem. Rev. 2016; 116: 9994-10034.

[23] Longevial JF, Clement S, Wytko JA, Ruppert R, Weiss J, Richeter S, Chem. Eur. J. 2018; 24: 15442-15460.

[24] Redmond RW, Gamlin JN, Photochem. Photobiol. 1999, 70: 391-475.

[25] Mathai S, Smith TA, Ghiggino KP, Photochem. Photobiol. Sci. 2007; 6: 995-1002.

[26] Ogoshi H, Mizutani T, Curr. Opin. Chem. Biol. 1999; 3: 736-739.

[27] Di Natale C, Martinelli E, Magna G, Mandoj F, Monti D, Nardis S, Stefanelli M,Paolesse R, J. Porphyr. Phthalocyanines 2017; 21: 769-781.

[28] Puigmartí-Luis J, Saletra WJ, González A, Amabilino D B, Pérez-García L, Chem. Commun. 2014; 50: 82-84.

[29] Antoni PM, Naik A, Albert I, Rubbiani R, Gupta S, Ruiz-Sanchez P, Munikorn P, Mateos JM, Luginbuehl V, Thamyongkit P, Ziegler U, Gasser G, Jeschke G, Spingler B, Chem. - A Eur. J. 2015; 21: 1179-1183.

[30] Fantuzzi G, Pengo P, Gomila R, Ballester P, Hunter CA, Itm CNR, Padova U, Uk S, Chem. Commun. 2003; 10041005.

[31] Figueira F, Cavaleiro JAS, Tomé JPCC, J. Porphyr. Phthalocyanines 2011; 15: 517-533.

[32] Yang CJ, Gao LC, Zhang BG, Zhang ZH, Deng KJ, J. Porphyr. Phthalocyanines 2018; 22: 868-876.

[33] Fernandez L, Esteves VI, Cunha A, Schneider RJ, Tomé JPCC, J. Porphyr. Phthalocyanines 2016; 20: $150-166$.

[34] Neves CMB, Filipe OMS; Mota N, Santos SAO, Silvestre AJD, Santos EBH, Neves MGPMS, Simoes MMQ, J. Hazardous Mater. 2018; https://doi.org/10.1016/j.jhazmat.2018.11.055 )

[35] Scanone AC, Gsponer NS, Alvarez MG, Durantini EN, J. Photochem. Photobiol. A Chem. 2017; 346: $452-461$.

[36] Torras N, Agusil JP, Vázquez P, Duch M, Hernández-Pinto AM, Samitier J, de la Rosa E J, Esteve J, Suárez T, Pérez-García L, Plaza, JA, Adv. Mater. 2016; 28: 1449-1454.

[37] Penon O, Marín M J, Amabilino D B, Russell DA, Pérez-García L, J. Colloid Interf. Sci. 2016, 462: $154-165$.

[38] Penon O, Marín M J, Russell DA, Pérez-García L, J. Colloid Interf. Sci. 2017, 496: 100-110.

[39] Alea-Reyes ME, Soriano J, Mora-Espí I, Rodrigues M, Russell DA, Barrios L, Pérez-García, L, Colloids Surf. B Biointerfaces 2017, 158: 602-609.

[40] Alea-Reyes ME, Penon O, García Calavia P, Marín M J, Russell DA, Pérez-García L, J. Colloid Interf. Sci. 2018, 521: $81-90$.

[41] Dumoulin F, Nano- and Molecular Engineering of Photosensitisers (Special issue), Photochem. Photobiol. Sci. $2018 ; 17: 1479-1806$.

[42] Agusil JP, Torras N, Duch M, Esteve J, Suárez T, Pérez-García L, Plaza, JA, Adv. Funct. Mater. 2017; 27: 16059121605923.

[43] Gómez-Martínez R, Vázquez P, Duch M, Muriano A, Pinacho D, Sanvicens N, Sánchez-Baeza F, Boya P, de la Rosa EJ, Esteve J, Suárez T, Plaza JA, Small 2010; 6: 499-502.

[44] Alea-Reyes ME, Rodrigues M, Serrà A, Mora M, Sagristá M L, González A, Durán S, Duch M, Plaza J A, Vallés E, Russell D A, Pérez-García L, RSC Advances 2017; 7:16963-16976.

[45] Okesola BO, Vieira VMP, Cornwell DJ, Whitelaw NK, Smith DK, Soft Matter 2015; 11: 4768-4787. 


\section{List of Captions}

Fig. 1. Polysilicon microparticles $(\boldsymbol{\mu} \mathbf{P})$ fabrication process. a) Silicon wafer as a substrate. b) Silicon oxide (sacrificial layer) deposition. c) Polysilicon (device layer) deposition. d) Photoresist deposition. e) Photolithographic step. f) Polysilicon dry etching (chips patterning). g) Photoresist removing. h) Sacrificial etching and chips release. Microscopy top views of the microparticles i) before and j) after release. Scale bar $5 \mu \mathrm{m}$

Fig. 2. Description and characterisation of the polysilicon microparticles $(\boldsymbol{\mu P})$. a) Representation showing the lateral dimensions of one individual $\mu \mathbf{P}$. b) Scanning electron microscopy image of the released $\mu \mathbf{P}$. c) Brightfield (left) and fluorescence (right) microscopy images of porphyrin labelled polysilicon hexahedrons (ITC-P- $\boldsymbol{\mu P}$ ). d) Representation of the cross-sections taken for the fluorescence intensity analysys and e) Profile of the fluorescence intensity on one ITC$\mathbf{P}-\mu \mathbf{P}$. f) Ideal representation of an overall porphyrin functionalized ITC-P- $\mu \mathbf{P}$

Fig. 3. Fluorescence intensity decay in the emission spectra of ADMA after irradiation: a) ADMA only in acetone, b) ADMA only in gel, c) ITC-P in acetone solution $(9 \mu \mathrm{M})$, d) TUEE-P in acetone solution $(9 \mu \mathrm{M})$, e) ITC-P- $\mu$ P (equivalent to ca $0.49 \mathrm{mM}$ of immobilized ITC-P) encapsulated in the supramolecular hydrogel

Fig. 4. Generation of singlet oxygen. a) Progressive decay of the ADBA emission band at $\lambda=430$ nm upon irradiation of samples ADMA only in acetone (purple), ADMA only in gel (green), ITC-P in acetone solution (orange), TUEE-P in acetone solution (blue), ITC-P- $\boldsymbol{\mu P}$ encapsulated in the supramolecular hydrogel (red). b) Percentage rate of ADMA photobleaching of the porphyrin in solution ITC-P and TUEE-P and functionalized ITC-P- $\mu$ P calculated based on the concentration of photosensitizer on the particles

Scheme 1. Stepwise Immobilization of ICT-P on $\mu \mathbf{P}$ to yield pophyrin functionalized ITC-P- $\mu \mathbf{P}$. 
a

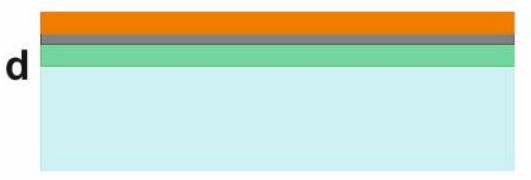

g
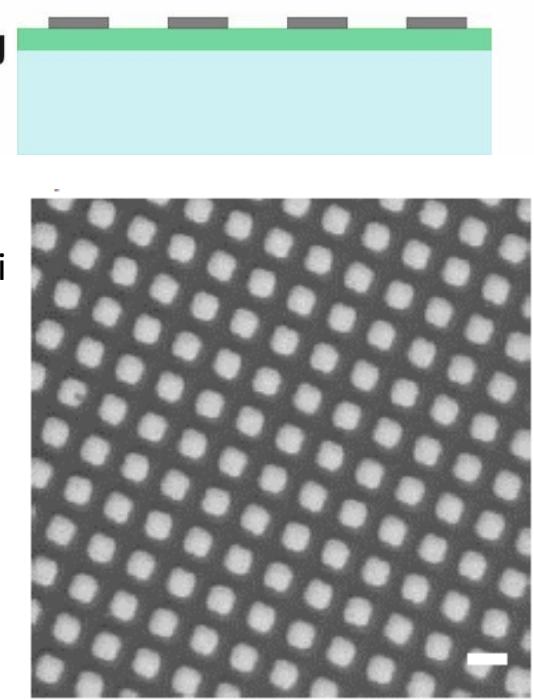

b

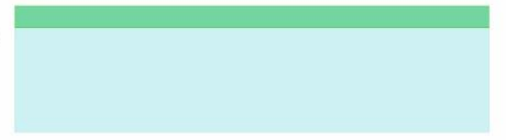

e

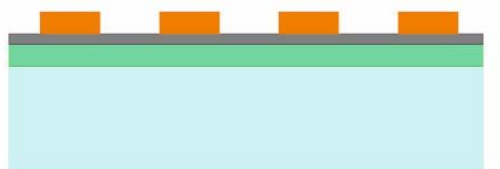

h
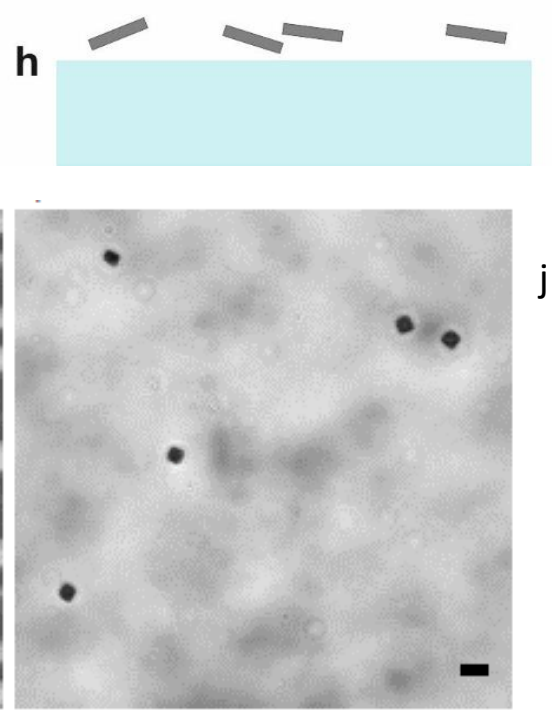

C

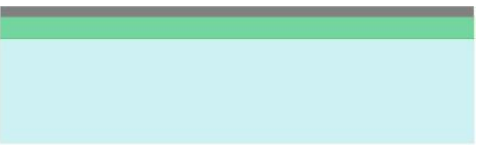

f

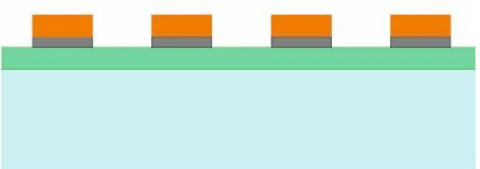

Silicon substrate

Silicon oxide

Polysilicon

Photoresist

Fig. 1. Polysilicon microparticles $(\boldsymbol{\mu P})$ fabrication process. a) Silicon wafer as a substrate. b) Silicon oxide (sacrificial layer) deposition. c) Polysilicon (device layer) deposition. d) Photoresist deposition. e) Photolithographic step. f) Polysilicon dry etching (chips patterning). g) Photoresist removing. h) Sacrificial etching and chips release. Microscopy top views of the microparticles i) before and j) after release. Scale bar $5 \mu \mathrm{m}$ 
a)

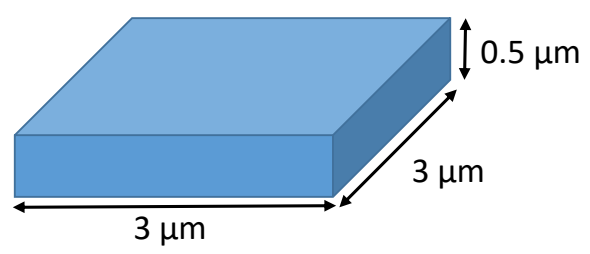

c)

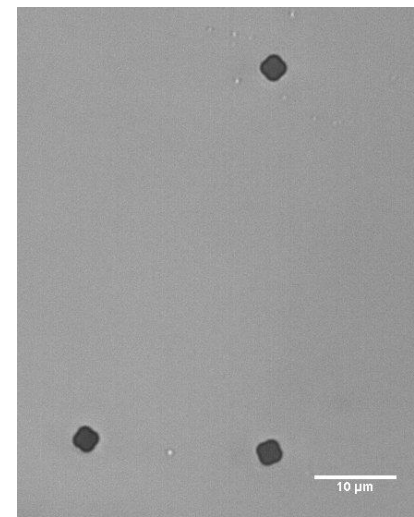

b)
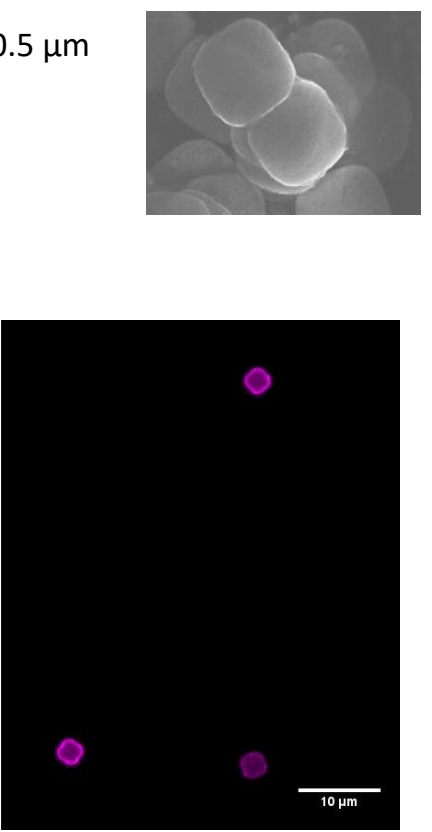

d)

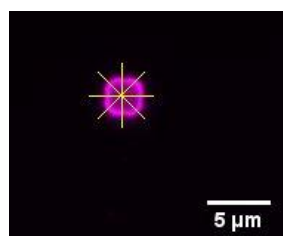

$5 \mu \mathrm{m}$

f)

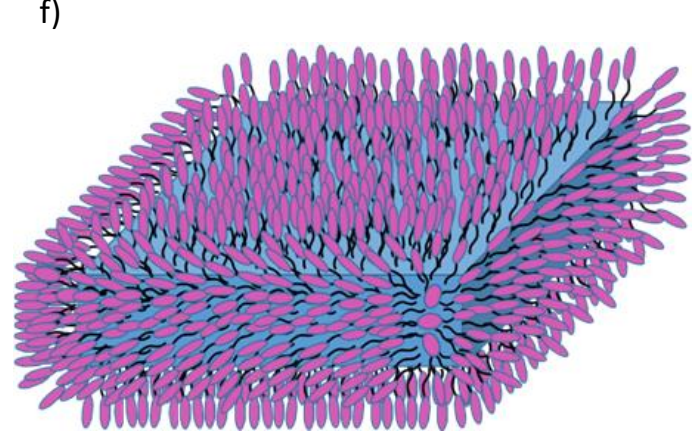

Fig. 2. Description and characterisation of the polysilicon microparticles $(\boldsymbol{\mu P})$. a) Representation showing the lateral dimensions of one individual $\boldsymbol{\mu P}$. b) Scanning electron microscopy image of the released $\boldsymbol{\mu P}$. c) Brightfield (left) and fluorescence (right) microscopy images of porphyrin labelled polysilicon hexahedrons (ITC-P- $\boldsymbol{\mu P}$ ). d) Representation of the cross-sections taken for the fluorescence intensity analysys and e) Profile of the fluorescence intensity on one ITC$\mathbf{P}-\mu \mathbf{P}$. f) Ideal representation of an overall porphyrin functionalized ITC-P- $\mu \mathbf{P}$ 
a)

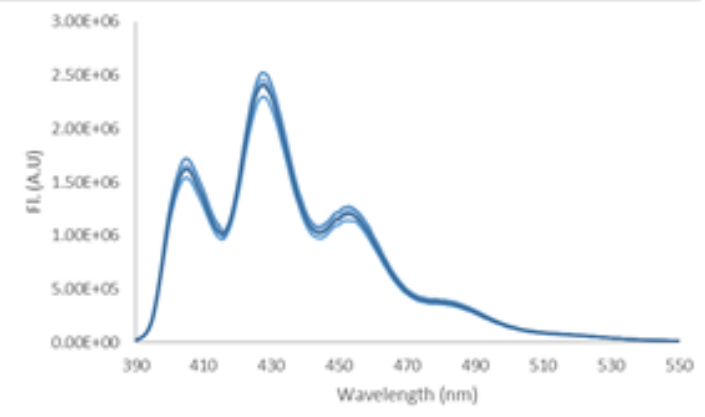

c)

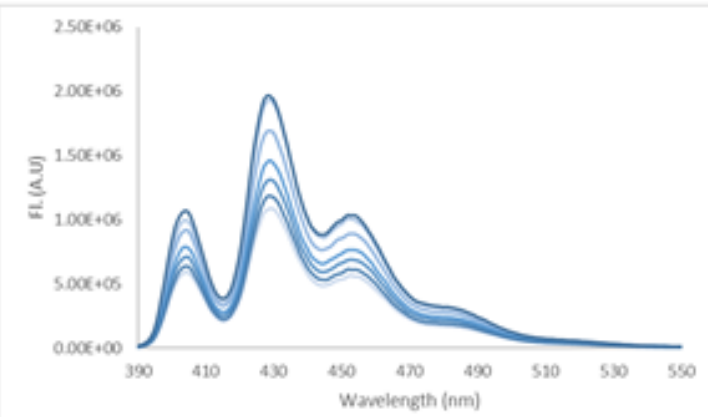

e)

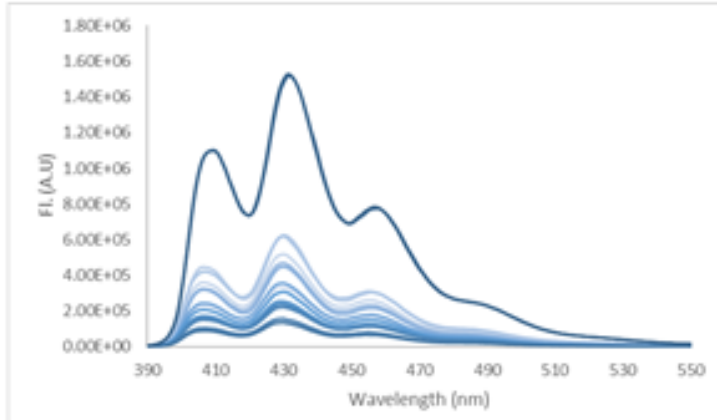

b)

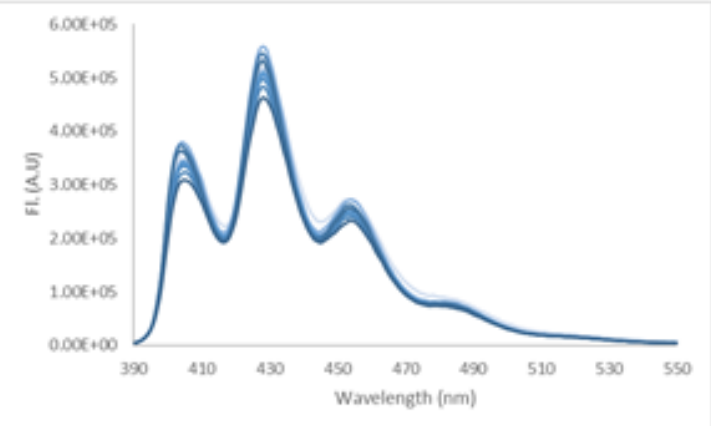

d)

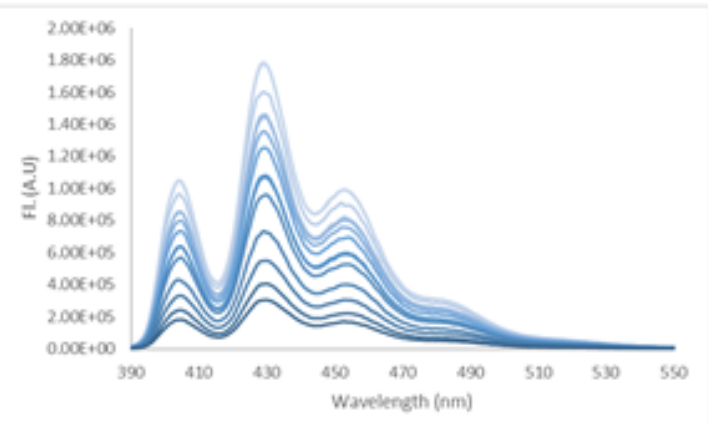

Fig. 3. Fluorescence intensity decay in the emission spectra of ADMA after irradiation: a) ADMA only in acetone, b) ADMA only in gel, c) ITC-P in acetone solution $(9 \mu \mathrm{M})$, d) TUEE-P in acetone solution $(9 \mu \mathrm{M})$, e) ITC-P- $\mu$ P (equivalent to ca $0.49 \mathrm{mM}$ of immobilized ITC-P) encapsulated in the supramolecular hydrogel 
a)
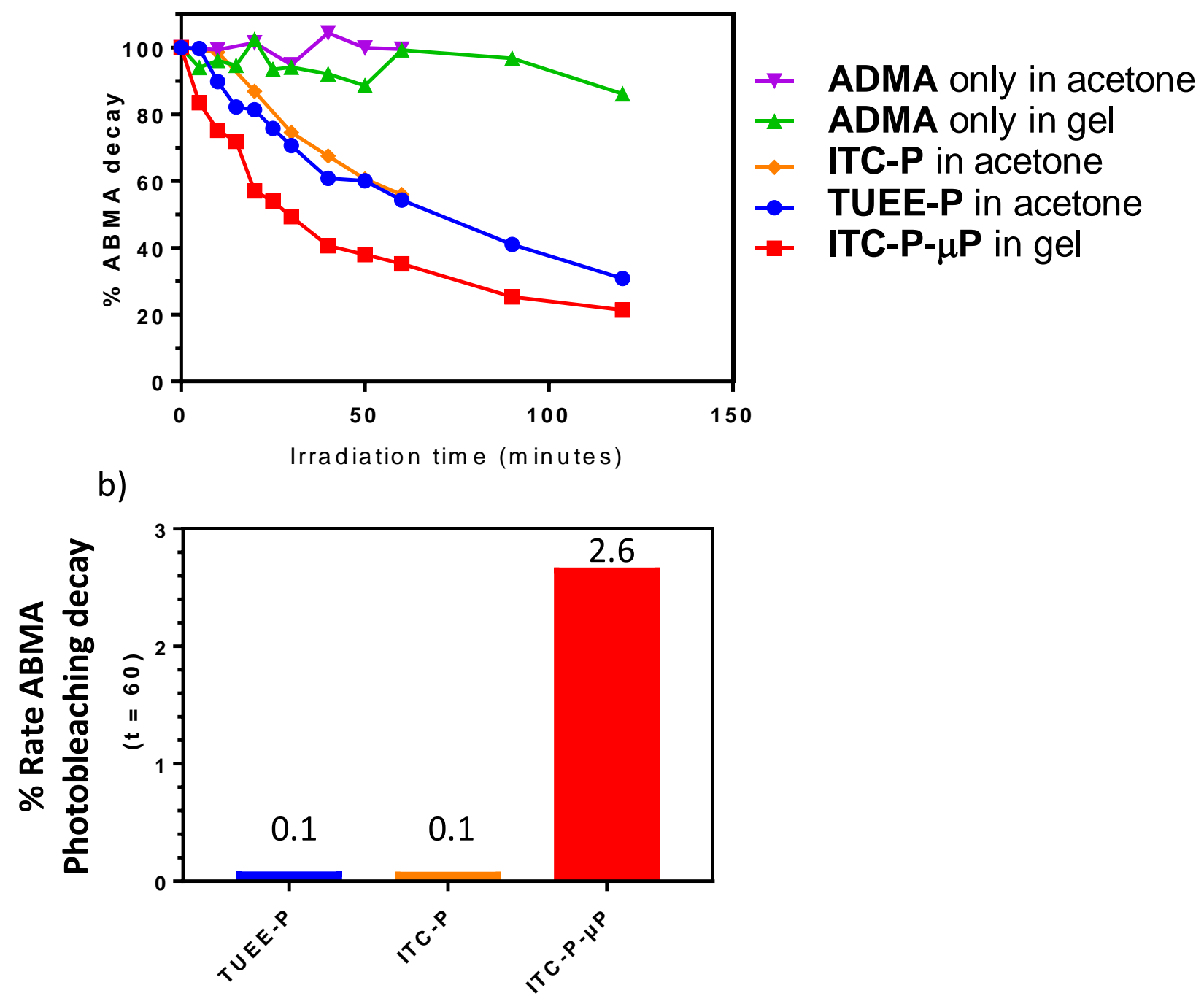

Fig. 4. Generation of singlet oxygen. a) Progressive decay of the ADBA emission band at $\lambda=430 \mathrm{~nm}$ upon irradiation of samples ADMA only in acetone (purple), ADMA only in gel (green), ITC-P in acetone solution (orange), TUEE-P in acetone solution (blue), ITC-P- $\mu$ P encapsulated in the supramolecular hydrogel (red). b) Percentage rate of ADMA photobleaching of the porphyrin in solution ITC-P and TUEE-P and functionalized ITC-P- $\mu \mathbf{P}$ calculated based on the concentration of photosensitizer on the particles 

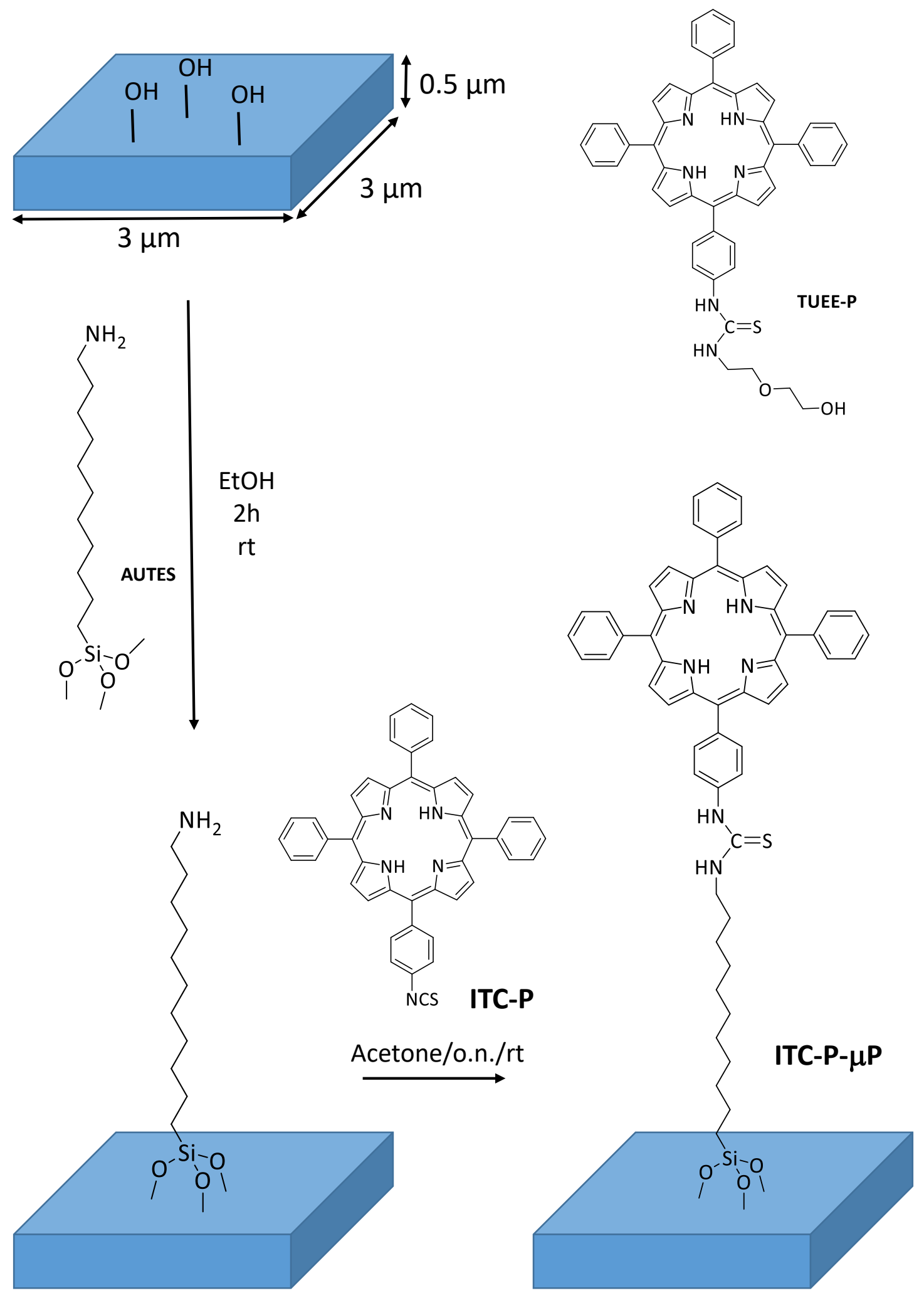

Scheme 1. Stepwise Immobilization of ICT-P on $\mu \mathbf{P}$ to yield pophyrin functionalized ITC-P- $\mu \mathbf{P}$ 